\title{
Smooth Model Surfaces from Lignin Derivatives. II. Adsorption of Polyelectrolytes and PECs Monitored by QCM-D
}

\author{
Magnus Norgren, ${ }^{*}{ }^{\dagger}$ Linda Gärdlund, ${ }^{\dagger}$ Shannon M. Notley, ${ }^{\ddagger}$ Myat Htun, ${ }^{\dagger}$ and Lars Wågberg ${ }^{\S}$ \\ Department of Natural Sciences, Fibre Science and Communication Network, Mid Sweden University, \\ SE-851 70 Sundsvall, Sweden, Department of Applied Mathematics, Research School of Physical Sciences \\ and Engineering, Australian National University, Canberra, ACT 0200, Australia, and Department of \\ Fibre and Polymer Technology, Royal Institute of Technology, SE-100 44 Stockholm, Sweden
}

Received November 27, 2006. In Final Form: January 12, 2007

\begin{abstract}
For the first time to the knowledge of the authors, well-defined and stable lignin model surfaces have been utilized as substrates in polyelectrolyte adsorption studies. The adsorption of polyallylamine (PAH), poly(acrylic acid) (PAA), and polyelectrolyte complexes (PECs) was monitored using quartz crystal microgravimetry with dissipation (QCMD). The PECs were prepared by mixing PAH and PAA at different ratios and sequences, creating both cationic and anionic PECs with different charge levels. The adsorption experiments were performed in 1 and $10 \mathrm{mM}$ sodium chloride solutions at $\mathrm{pH} 5$ and 7.5. The highest adsorption of PAH and cationic PECs was found at $\mathrm{pH} 7.5$, where the slightly negatively charged nature of the lignin substrate is more pronounced, governing electrostatic attraction of oppositely charged polymeric substances. An increase in the adsorption was further found when the electrolyte concentration was increased. In comparison, both PAA and the anionic PEC showed remarkably high adsorption to the lignin model film. The adsorption of PAA was further studied on silica and was found to be relatively low even at high electrolyte concentrations. This indicated that the high PAA adsorption on the lignin films was not induced by a decreased solubility of the anionic polyelectrolyte. The high levels of adsorption on lignin model surfaces found both for PAA and the anionic PAA-PAH polyelectrolyte complex points to the presence of strong nonionic interactions in these systems.
\end{abstract}

\section{Introduction}

Lignins belong to a group of natural polymers that are based on three main monomers of phenyl propanoic origin linked together in about ten different ways creating a somewhat crosslinked chemical structure. ${ }^{1}$ Lignins are found in trees and many 1 year crops and provide mechanical strength and water resistance to the organism. Today the chemistry of lignin is quite well understood and the efforts of elucidating the biosynthetic pathways are progressing well. ${ }^{2}$ The separation of lignins from woody materials renders relatively complex and heterogeneous chemical structures as well as a polydisperse and noncrystalline material. ${ }^{3}$ For instance in the kraft process, the wood is treated with a cooking liquor containing approximately $0.2 \mathrm{M}$ sodium sulfide and $1 \mathrm{M}$ sodium hydroxide at $150-170{ }^{\circ} \mathrm{C}$ for more than $1 \mathrm{~h}$. This induces depolymerization of the lignin structure by cleavage of aryl-ether bonds and an increased number of free phenols and to some extent also carboxylic groups. ${ }^{1}$ Therefore the use of lignin has been mostly restricted to relatively simple additives, e.g., in concrete and in oil recovery.

Even though lignins are the second most abundant group of biopolymers, physicochemical interactions involving lignin in pulp and paper science have received rather low attention in the past. When manufacturing paper, the molecular interactions taking place in the contact zone between the fibers are certainly important. ${ }^{4-8}$ In recent years, model studies of cellulose-cellulose

* Corresponding author. E-mail: magnus.norgren@miun.se. Phone: +46 60 148877. Fax: +4660148820.

Mid Sweden University.

$\doteqdot$ Australian National University.

$\S$ Royal Institute of Technology.

(1) Sjöström, E. Wood Chemistry. Fundamentals and Applications, 2nd ed.; Academic Press, Inc.: San Diego, 1993.

(2) Li, L. G.; Lu, S. F.; Chiang, V. Crit. Rev. Plant Sci. 2006, 25, 215

(3) Goring, D. A. I. Pure Appl. Chem. 1962, 5, 233.

(4) Torgnysdotter, A.; Wågberg, L. Nordic Pulp Paper Res. J. 2003, 18, 455.

(5) Torgnysdotter, A.; Wågberg, L. Nordic Pulp Paper Res. J. 2004, 19, 440. interactions or cellulose's interactions with paper chemicals and inorganic particles have been focused in several investigations. ${ }^{9-17}$ Here, the development of well-defined cellulose model films has been a necessity in order to make fundamental investigations possible. This is gladly a huge step forward in the field. However, in our efforts to understand fiber-fiber interactions on a molecular level, well-defined model systems including other polysaccharides and of course stable lignin model surfaces are still required.

Depending on the wood species as well as the pulping and bleaching processes, the lignin content of the pulp fibers varies from zero to about thirty percent. A somewhat higher surface lignin concentration compared to the bulk of the fiber can be observed in some cases. ${ }^{18}$ Nevertheless, in many pulp qualities, lignin at the surfaces of the fibers undoubtedly must interact with other wood constituents as well as with various paper additives. Until today, numerous investigations where lignin films have been prepared on various substrates from different lignins

(6) Gärdlund, L.; Forsström, J.; Andreasson, B.; Wågberg, L. Nordic Pulp Paper Res. J. 2005, 20, 36.

(7) Forsström, J.; Torgnysdotter, A.; Wågberg, L. Nordic Pulp Paper Res. J. 2005, 20, 186.

(8) Pettersson, G.; Höglund, H.; Wågberg, L. Nordic Pulp Paper Res. J. 2006, $21,122$.

(9) Notley, S. M.; Petterson, B.; Wågberg, L. J. Am. Chem. Soc. 2004, 126, 13930

(10) Notley, S. M.; Wågberg, L. Biomacromolecules 2005, 6, 1586.

(11) Geffroy, C.; Labeau, M. P.; Wong, K.; Cabane, B.; Cohen-Stuart, M. A. Colloids Surf. A 2000, 172, 47.

(12) Poptoshev, E.; Claesson, P. M. Langmuir 2002, 18, 1184

(13) Lefebvre, J.; Gray, D. G. Cellulose 2005, 12, 127.

(14) Radtchenko, I. L.; Papastavrou, G.; Borkovec, M. Biomacromolecules 2005, 6, 3057.

(15) Forsström, J.; Eriksson, M.; Wågberg, L. J. Adhesion Sci. Technol. 2005, 19,783 .

(16) Notley, S. M.; Eriksson, M.; Wågberg, L.; Beck, S.; Gray, D. G. Langmuir 2006, 22, 3154.

(17) Theander, K.; Pugh, R. J.; Rutland, M. W. J. Colloid Interface Sci. 2005 291,361

(18) Sjöberg, J.; Kleen, M.; Dahlman, O.; Agnemo, R.; Fabriker, D.; Sundvall, H. Nordic Pulp Paper Res. J. 2002, 17, 295. 
and by various methods have been reported. ${ }^{19-31}$ Despite that, the use of lignin model surfaces in further fundamental experiments has been limited. Partly, this could be due to problems related to high surface roughness due to extensive particle formation or inhomogeneous substrate coverage. The latter might for instance be influenced by the choice of solvent, which could generate only partial dissolution of the lignin, low wetting and spreading of the substrate or too rapid evaporation that creates pores or depleted regions in the films produced. Instability of the model surface due to low adhesion to the substrate, or simply by dissolution of the film, could induce severe problems in further experiments. Moreover, tedious and complicated preparation methods could also provide certain limitations. Recently, a protocol for the preparation of well-defined lignin model surfaces of very low roughness by spin-coating on silica wafers was presented. ${ }^{30}$ Spin-coating is a useful method for preparation of thin, uniform films on flat substrates. An excess amount of polymer solution is placed on the substrate. The substrate is then rotated at high speed in order to spread the fluid and thereafter dry the film. By dissolving kraft lignin in aqueous ammonium hydroxide to a concentration of between 0.5 and $3.0 \mathrm{wt} \%$, lignin films of thicknesses between 20 and $140 \mathrm{~nm}$ could be prepared successfully. The stability of these lignin model surfaces in Milli-Q water was very good even after days of soaking. In a slightly alkaline aqueous environment, the lignin films showed no signs of deterioration below $\mathrm{pH} 9$ even after several hours. These results open up great new possibilities for studying fundamental interactions between solid lignin and other substances by various experimental techniques.

Cationic polyions are regularly used as strength additives in papermaking. Due to the anionic nature of the cellulose and other woody components, the electrostatic interactions between cationic additives and the fiber material are obvious and very important for the development of paper strength. A different but very promising approach to achieve a large spatial extension of the strength additive is to prepare polyelectrolyte complexes (PECs) consisting of a tuned mixture of negatively and positively charged polyelectrolytes respectively. ${ }^{32-34}$ The fundamentals of formation of PECs by combining various polyelectrolytes have also been earlier described in the literature. ${ }^{35,36}$

(19) Lee, S. B.; Luner, P. Tappi J. 1972, 55, 116.

(20) Micic, M.; Radotic, K.; Benitez, K.; Ruano, M.; Jeremic, M.; Moy, V.; Mabrouki, M.; Leblanc, R. M. Biophys. Chem. 2001, 94, 257.

(21) Micic, M.; Benitez, I.; Ruano, M.; Mavers, M.; Jeremic, M.; Radotic, K.; Moy, V.; Leblanc, R. M. Chem. Phys. Lett. 2001, 347, 41.

(22) Micic, M.; Jeremic, M.; Radotic, K.; Mavers, M.; Leblanc, R. M. Scanning 2000, 22, 288.

(23) Micic, M.; Radotic, K, Jeremic, M.; Djikanovic, D.; Kämmer, S. B. Colloids Surf. B 2004, 34, 33.

(24) Constantino, C. J. L.; Juliani, L. P.; Botaro, V. R.; Balogh, D. T.; Pereira, M. R.; Ticianelli, E. A.; Curvelo, A. A. S.; Oliveira, O. N., Jr. Thin Solid Films 1996, 284-285, 191

(25) Constantino, C. J. L.; Dhanabalan, A.; Curvelo, A. A. S.; Oliveira, O. N., Jr. Thin Solid Films 1998, 327-329, 47.

(26) Constantino, C.; Dhanabalan, A.; Cotta, M.; Pereira-da-Silva Curvelo, A.; Oliveira, O., Jr. Holzforschung 2000, 54, 55.

(27) Pasquini, D.; Balogh, D. T.; Antunes, P. A.; Constantino, C. J. L.; Curvelo,

A. A. S.; Aroca, R. F.; Oliveira, O. N., Jr. Langmuir 2002, 18, 6593.

(28) Pasquini, D.; Balogh, D. T.; Olivera, O. N., Jr.; Curvelo, A. A. S. Colloids

Surf. A 2005, 252, 193.

(29) Paterno, L. G.; Mattoso, L. H. C. Polymer 2001, 42, 5239.

(30) Norgren, M.; Notley, S. M.; Majtnerova, A.; Gellerstedt, G. Langmuir 2006, 22, 1209.

(31) Maximova, N.; Österberg, M.; Laine, J.; Stenius, P. Colloids Surf. A 2004, 239, 65 .

(32) Gernandt, R.; Wågberg, L.; Gärdlund, L.; Dautzenberg, H. Colloids Surf A 2003, 213, 15 .

(33) Gärdlund, L.; Wågberg, L.; Gernandt, R. Colloids Surf. A 2003, 218, 137.

(34) Gärdlund, L.; Forsström, J.; Andreasson, B.; Wågberg, L. Nordic Pulp Paper Sci. 2005, 20, 36.

(35) Tsuchida, E.; Abe, K. Adv. Polym. Sci. 1982, 45, 1.

(36) Dautzenberg, H. In Physical chemistry of polyelectrolytes; Radeva, T., Ed., CRC Press: New York, 2001; pp 743-792.

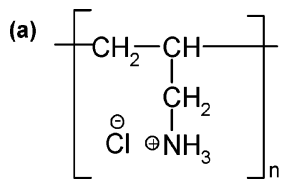

(b)<smiles>CC(C)(C)CC(C(=O)O)C(C)(C)C</smiles>

Figure 1. Chemical structures of (a) polyallylamine hydrchloride (PAH) and (b) poly(acrylic acid) (PAA).

The present paper reports the results from an adsorption study of PECs on lignin model films and silica surfaces. Various mixing ratios and combinations of polyallylamine hydrochloride (PAH) and poly(acrylic acid) (PAA) as well as the polyelectrolyte constituents themselves have been investigated. The adsorption was monitored by quartz crystal microgravimetry with dissipation (QCM-D) over a range of $\mathrm{pH}$-values and ionic strengths.

\section{Experimental Section}

Materials. The lignin derivative used was a softwood kraft lignin obtained by the following isolation and purification procedure. To $100 \mathrm{~mL}$ of kraft cooking liquor (softwood pulp of kappa number 24.7) was added EDTA-disodium ( $1 \mathrm{~g}$ in $80 \mathrm{~mL}$ of water) to chelate any present metal ions. Then the liquor was neutralized with sulfuric acid to $\mathrm{pH}=5.05\left(0.5 \mathrm{M} \mathrm{H}_{2} \mathrm{SO}_{4}\right)$ while gaseous nitrogen was bubbling through the liquor in order to remove the malodorous gases. The combined precipitate and solution was frozen overnight. After towing to $0{ }^{\circ} \mathrm{C}$ the lignin precipitate was collected on a glass filter $-\mathrm{G} 4$ and washed with small amount of icy water. The lignin was suspended in water and freeze-dried. Subsequently the lignin was extracted with pentane for $8 \mathrm{~h}$ to remove extractives. In order to remove carbohydrates the lignin was suspended in dioxane:water (9:1) at room temperature and stirred for $2 \mathrm{~h}$. After that the solution was centrifuged and the residue was repetitively treated with dioxane: water until the solution remained colorless. After evaporation of the dioxane, the suspension of lignin was freeze-dried. In order to remove the low molecular weight fraction from the lignin, the isolated lignin was stirred in dichloromethane for $2 \mathrm{~h}$ at room temperature (approximately $1 \mathrm{~g}$ of lignin per $100 \mathrm{~mL}$ of $\mathrm{CH}_{2} \mathrm{Cl}_{2}$ ). The lignin was filtered through a glass filter and washed with $\mathrm{CH}_{2} \mathrm{Cl}_{2}$ until the filtrate remained colorless. The insoluble lignin was collected and dried. According to pulsed field gradient ${ }^{1} \mathrm{H}$ NMR self-diffusion measurements, the kraft lignin had a weight averaged molecular mass, $M_{\mathrm{w}}$, of $5670 \mathrm{~g} \mathrm{~mol}^{-1}$ and the polydispersity index, $M_{\mathrm{w}} / M_{\mathrm{n}}$, was 1.54 .

The cationic polyelectrolyte used in this study was a polyallylamine hydrochloride $(\mathrm{PAH}), M_{\mathrm{w}}=70000 \mathrm{~g} \mathrm{~mol}^{-1}$, and the anionic polyelectrolyte was poly(acrylic acid) (PAA) in sodium form, $M_{\mathrm{w}}$ $=8000 \mathrm{~g} \mathrm{~mol}^{-1}$ (see Figure 1). The polyelectrolytes were purchased from Aldrich Chemicals (Sigma-Aldrich), and the molecular masses were given by the supplier. The water used for preparation of the complexes was of Milli-Q grade. Prior to complex formation the charge of the polyelectrolytes was determined at $\mathrm{pH}$ 7. This was performed by titrating the polycation and polyanion with potassium polyvinylsulfate (KPVS) and poly dimethyldiallyl ammonium chloride (PDMDAAC), respectively. The point of charge reversal was detected with a streaming current detector, Mütec PCD 03 (Mütec, Germany). The PAH had a charge density of 8.9 mequiv $\mathrm{g}^{-1}$, the PAA 10.4 mequiv $\mathrm{g}^{-1}$. These values were used when preparing the different charge molar mixing ratios of the PECs.

Methods. Quartz. Crystal Microgravimetry with Dissipation Monitoring ( $Q C M-D)$. The adsorption experiments were performed using a Q-Sense D300 instrument (Q-Sense AB, Sweden), which measures both the frequency and the dissipation simultaneously. 5 MHz-AT cut sensor crystals with $\mathrm{SiO}_{2}$ coatings were used with and without spin-coated lignin films. In all measurements, the cell was initially filled with Milli-Q water and rinsed a couple of times until a stable baseline was established. Data were collected using Q-Soft (Q-Sense AB, Sweden) at the fundamental frequency $(5 \mathrm{MHz})$ and at the third and fifth overtones (15 and $25 \mathrm{MHz}$ ), but the third overtone has primarily been used in the data evaluation. The temperature in the QCM-D measuring chamber during 
the adsorption experiments was carefully controlled and set to $23{ }^{\circ} \mathrm{C}$. The third overtone was used to determine the adsorbed mass as the data collected from the fundamental frequency of this instrument is somewhat unstable due to energy trapping by the O-ring.

Spin-Coating. A Chemat Technology KW-4A spin-coater (Chemat Technology, England) was used to coat the QCM crystals. Before the spin-coating, the QCM crystals were exposed to $0.1 \mathrm{M} \mathrm{NaOH}$ three times for $20 \mathrm{~s}$, rinsed with plenty of Milli-Q water, and dried by a stream of nitrogen gas. In the preparation of the lignin films a speed of $2000 \mathrm{rpm}$ for $1 \mathrm{~min}$ was applied and the lignin concentration was $2.0 \mathrm{wt} \%$ in $1.0 \mathrm{M} \mathrm{NH}_{3}$.

Atomic Force Microscopy (AFM). The prepared lignin surfaces were imaged with tapping-mode atomic force microscopy (Nanoscope III, Multimode SPM, Veeco Inc.) to determine the surface roughness and morphology. The images were recorded in ambient air conditions $\left(25^{\circ} \mathrm{C}\right.$ and $50 \%$ relative humidity) with a TESP, rectangular silica cantilever (Veeco, Inc.).

Preparation of Polyelectrolyte Complexes (PECs). The polycation was diluted to 1 mequiv $\mathrm{L}^{-1}$ at $\mathrm{pH} 7$, the polyanion to 2 mequiv $\mathrm{L}^{-1}$ also at $\mathrm{pH}$ 7. The $\mathrm{pH}$ was adjusted using $\mathrm{NaOH}$ and $\mathrm{HCl}$. Stirring of the polycation was continued, and the polyanion was added to the polycation with the aid of an automatic titrator, Titrino SM 702 (Metrohm, Switzerland), under continuous stirring of the starting solution. The cationic complex solutions were prepared at molar mixing ratios $(\mathrm{q}-/ \mathrm{q}+)$ of 0.8 and 0.3 , respectively. Cationic complex solutions of a molar mixing ratio of 0.8 were prepared by adding $60 \mathrm{~mL}$ of the polyanion solution to $150 \mathrm{~mL}$ of the polycation solution. At molar mixing ratios of 0.3 , PECs were formed by adding 22.5 $\mathrm{mL}$ of the polyanion solution to $150 \mathrm{~mL}$ of the polycation starting solution. The dosage rate was $0.5 \mathrm{~mL} \mathrm{~min}{ }^{-1}$ in both the cases. The formed PEC solutions were gently stirred and used within $72 \mathrm{~h}$. The anionic PECs were prepared by the same method, but with reversed mixing order of the polyelectrolytes. The PEC solutions were diluted to $50 \mathrm{mg} \mathrm{L}^{-1}$ and $\mathrm{pH}$ was adjusted prior to adsorption measurements in the QCM.

Dynamic Light Scattering Measurements. A Brookhaven 90 Plus particle size analyzer (Brookhaven Instruments Corp.) equipped with a $30 \mathrm{~mW}$ solid-state laser was used to determine the hydrodynamic radii of the PECs. To avoid hindered self-diffusion, the PEC concentration in the sample cell was held low, typically at around $3 \mathrm{mg} \mathrm{L}^{-1}$.

Cryogenic Transmission Electron Microscopy (cryo-TEM). A Philips CM120 BioTWIN transmission electron microscope specially designed for cryo measurements and equipped with a Gatan energy filter GIF 100 was used. The microscope was operating at $120 \mathrm{kV}$ in low dose mode and the micrographs were taken utilizing the zero loss peak at a slit width of $8 \mathrm{eV}$. The cryoholder was an Oxford CT 3500 , and the samples were plunged into liquid ethane. ${ }^{37,38}$ The samples were always kept below $-180{ }^{\circ} \mathrm{C}$.

\section{Results and Discussion}

Stability of the Lignin Film in QCM-D Experiments. In Figure 2, a typical AFM image of a spin-coated QCM-D crystal is shown. An almost uniform coverage of somewhat spherical aggregates is observed in these images, typically with a diameter of $10-20 \mathrm{~nm} .{ }^{30}$ The preparation method has been shown to give rather low surface roughnesses and stable lignin model films in aqueous environments. This is also a prerequisite for meaningful use of these films in QCM-D studies.

The principle of QCM-D is to precisely measure the resonant frequency of a piezoelectric crystal. In the simple Sauerbrey

(37) Bellare, J. R.; Davis, H. T.; Scriven, L. E.; Talmon, Y. J. J. Electron Microsc. Tech. 1988, 10, 87 .

(38) Vinson, P. K. In The 45th Annual Meeting of the Electron Microscopy Society of America; San Francisco Press: San Francisco, 1987; pp 644-645.

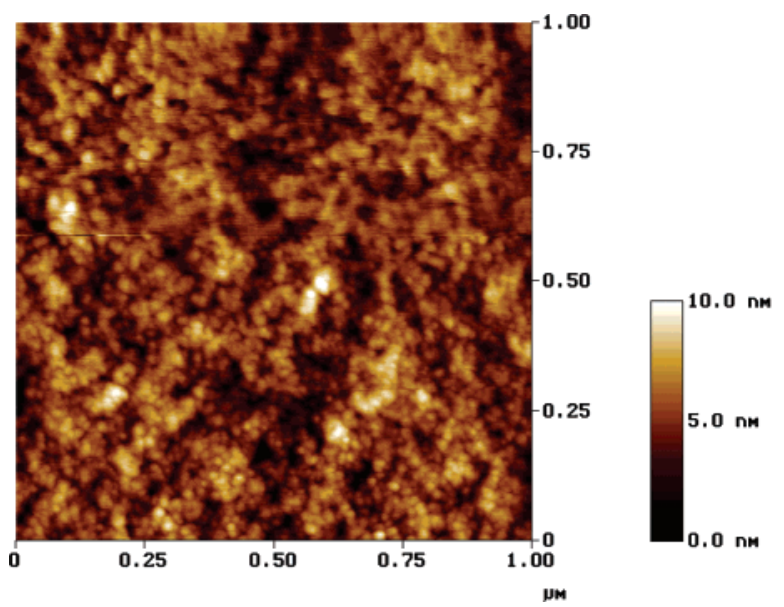

Figure 2. An AFM tapping-mode image of a lignin film spincoated on a silica sputtered QCM crystal. The smoothness, as expressed by the rms-value from the height image, was $1.4 \mathrm{~nm}$ at $1 \mu \mathrm{m}^{2}$ as determined from an average of 4 images.

model for mass determination, ${ }^{39}$ the adsorbed mass $\Delta m$ is proportional to the change in resonant frequency $\Delta f$ as

$$
\Delta m=C \frac{\Delta f}{n}
$$

Here $n$ is the number of the overtone and $C$ is a sensitivity constant. Equation 1 is assumed to give quantitatively correct values if the adsorbed layers are thin and rigidly attached to the surface. This adsorbed mass is composed both of the adsorbed amount of polymer and of the liquid immobilized in the adsorbed layer. For thicker layers that are less rigid, the direct proportionality in eq 1 breaks down, resulting in an underestimation of the adsorbed mass. ${ }^{40}$ The dissipation factor, $D$, is experimentally determined by measuring the time constant, $\tau$, for the exponential decay in the amplitude of the piezoelectric crystal when the driving voltage is turned off and is calculated as

$$
D=\frac{1}{\pi f \tau}
$$

For a studied system an increase in $D$ can be interpreted by means of an increased viscoelasticity of the adsorbed layer due to swelling, i.e., incorporation of water in the adsorbing substance. In contrast to adsorption studies, the QCM-D technique can also be used the opposite way to study desorption of coated layers from the crystal.

The stability of the lignin model film in the QCM-D measuring chamber was investigated over a wide $\mathrm{pH}$-range and at various ionic strengths to clearly define under which solution conditions reliable measurements could be performed. Figure 3 shows the response from the QCM-D when a lignin coated crystal is rinsed with aqueous solutions of gradually increasing sodium hydroxide concentrations. The resonance frequency remains stable as well as the dissipation of the crystal in the $\mathrm{pH}$-range 7-9. This indicates that the spin-coated lignin layer is intact and no changes due to swelling or deterioration of the lignin film are observed. When the $\mathrm{pH}$-value is raised to $\mathrm{pH} 9.5$, a minor decrease in $\Delta f$ and increase in $\Delta D$ can be detected. This is most probably due to a slight swelling of the lignin film on the crystal. At slightly higher alkalinity, $\mathrm{pH} 10, \Delta f$ is rapidly increasing along with the dissipation. This can only be interpreted as deterioration of the

(39) Sauerbrey, G. Z. Phys. 1959, 155, 206. 835. 

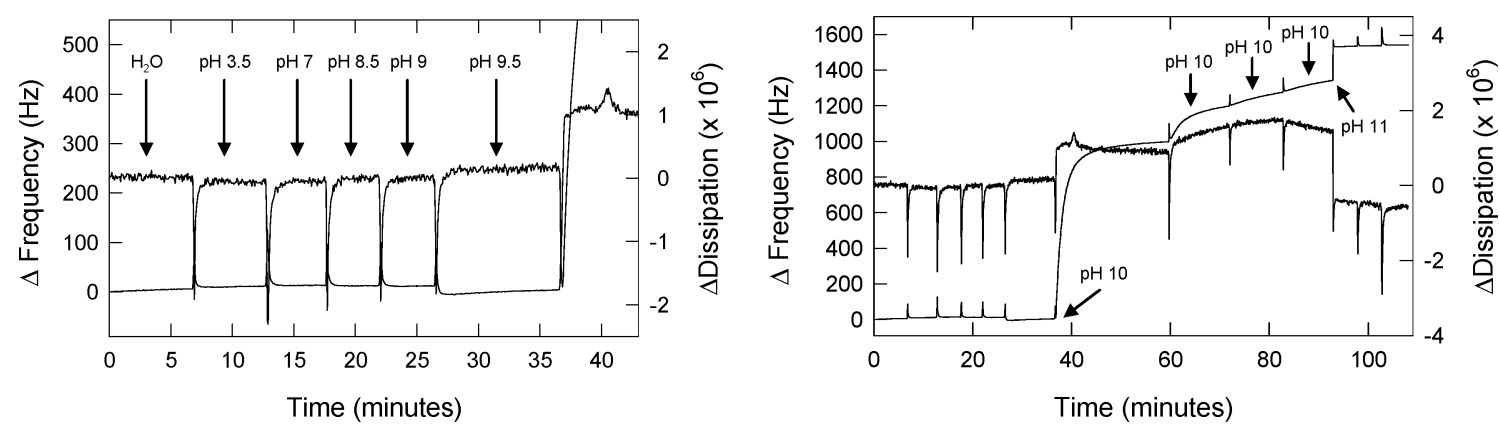

Figure 3. The effect of alkalinity on the stability of a spin-coated lignin film as monitored by QCM-D. The frequency curve is represented by the thin line. The arrows (left, pointing to the dissipation curve; right, pointing to the frequency curve) indicate the characteristics of the rinsing fluid introduced into the measuring chamber.

lignin film by desorption/dissolution of the solid lignin. The increase in dissipation suggests that some of the dissolved lignin is still loosely attached to the QCM-D crystal. During the following three rinses with the $\mathrm{pH} 10$ solution, the lignin is gradually desorbed according to the increase in resonance frequency. After the third rinse with the $\mathrm{pH} 10$ solution, a slight decrease in $\Delta D$, which indicates complete desorption of some of the loosely attached lignin in the film, is observed. Increasing the $\mathrm{pH}$ to $11 \mathrm{immediately}$ detaches the remaining lignin from the crystal as is illustrated by the "step-function behavior" of both the resonance frequency signal and the dissipation, followed by constant signal levels over time. The complete removal of the lignin film was further confirmed through naked-eye inspection of the QCM-D crystal after the experiment, showing a clean silica surface.

In order to compare the amount of lignin that was removed from the piezoelectric crystal in Figure 2 with what was expected to be put on by spin-coating at the conditions applied, eq 1 was used assuming a sensitivity constant, $C$, of $-0.177 \mathrm{mg} \mathrm{m}^{-2} \mathrm{~Hz}^{41}$ The observed frequency shift during the time of experiment in Figure 2 was $\Delta f=-1528 \mathrm{~Hz}$, which corresponds to a lignin mass loss of $8.63 \mu \mathrm{g}$ from the crystal surface. The active area of the crystal was $7.85 \times 10^{-5} \mathrm{~m}^{2}$ and the thickness of the lignin film before the experiment was determined to be within the interval $78-82 \mathrm{~nm}$ depending on the assumed density $(1.333-$ $\left.1.4 \mathrm{~g} \mathrm{~mL}^{-1}\right)^{42,43}$ of the lignin used. From the previous part of this work a lignin film thickness of approximately $80 \mathrm{~nm}$ would be expected at the present lignin concentration and spinning rate used for the spin-coater. ${ }^{30}$ The very good quantitative correspondence between the ellipsometry measurements of the lignin film thickness in the preparation study presented earlier and the results from the present QCM-D measurements indicates that the lignin model surfaces can be characterized as dense, rigid and poorly swollen, still following the Saurebrey relationship (eq 1) at fairly high film thicknesses.

Generally, the adsorption of charged polymers shows high sensitivity to the ionic strength of the solution due to variations in the electrostatic interactions within the polyion chain, between the polyelectrolytes and between charged polymers and substrates. Thus, in studies of polyelectrolyte adsorption, it must be possible to change the electrolyte concentration in the system to identify an overall picture of the process. This also makes heavy demands on the stability of the substrates, especially when it comes to films of spin-coated polyelectrolytes, which for example may shrink or swell uncontrollably, detach or dissolve in the presence of salt. Figure 4 shows the behavior of the lignin model film

(41) Edvardsson, M.; Rodahl, M.; Kasemo, B.; Höök, F. Anal. Chem. 2005 , 77,4918

(42) Ramaih, M. V.; Goring, D. A. I. J. Polym. Sci. C 1965, 11, 27.

(43) Stamm, A. J. Tappi J. 1969, 52, 1498.

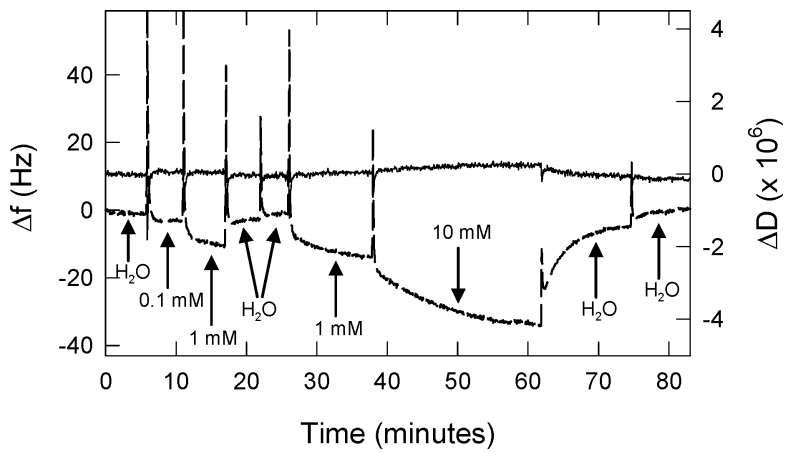

Figure 4. The QCM-D signal from an investigation of the effects of sodium chloride concentration on the lignin model surface stability. The given solution conditions in the measuring chamber are indicated on the frequency curve by the arrows.

under the influence of different solutions of various sodium chloride concentrations. It can be seen that the resonance frequency of the QCM crystal shifts to lower values when the electrolyte solutions are introduced into the sample chamber. This is probably due to several different phenomena occurring simultaneously. First, the density and viscosity of the electrolyte solutions are higher than those of pure deionized water, which results in a decrease in the resonance frequency of the QCM-D crystal. Moreover, a slight swelling of the film or ion adsorption may also be attributed. ${ }^{44}$ However, after rinsing the sample chamber with pure water, $\Delta f$ always returns to zero quite rapidly. The dissipation curve shows very small changes regardless of the concentration of the electrolyte solutions tested. This indicates that the changes of the lignin film are relatively small and reversible within the electrolyte concentration range investigated.

Adsorption of Cationic PECs and PAH. Figure 5 shows the adsorption of a cationic PEC of molar mixing ratio +0.3 (to 10 charge equivalents of PAH, 3 charge equivalents of PAA are added) at $\mathrm{pH} 7.5$ and $1 \mathrm{mM} \mathrm{NaCl}$. After 38 min the adsorption of the PEC had reached its maximum and the sample chamber was gently rinsed with water whereas the resonance frequency increased slightly and finally settled at $\Delta f=-20 \mathrm{~Hz}$. By applying eq 1 in Figure 5 the amount of cationic PECs adsorbed is around $1.4 \mathrm{mg} \mathrm{m}^{-2}$ including immobilized water and the change in dissipation approximately $0.1 \times 10^{-6}$, indicating that the PECs are adsorbed as relatively compact particles.

A comparison between the adsorption of the cationic PAHPAA PEC and its cationic constituent was further performed. In Figure 6 is shown the result of a QCM-D experiment where PAH was allowed to adsorb on a lignin-coated crystal at $\mathrm{pH} 7.5$ and $1 \mathrm{mM} \mathrm{NaCl}$. After approximately $33 \mathrm{~min}$, the sample chamber was rinsed with water. The adsorbed $\mathrm{PAH}$ layer at the end of

(44) Kanazawa, K. K.; Gordon, II J. G. Anal. Chim. Acta 1985, 175, 99. 


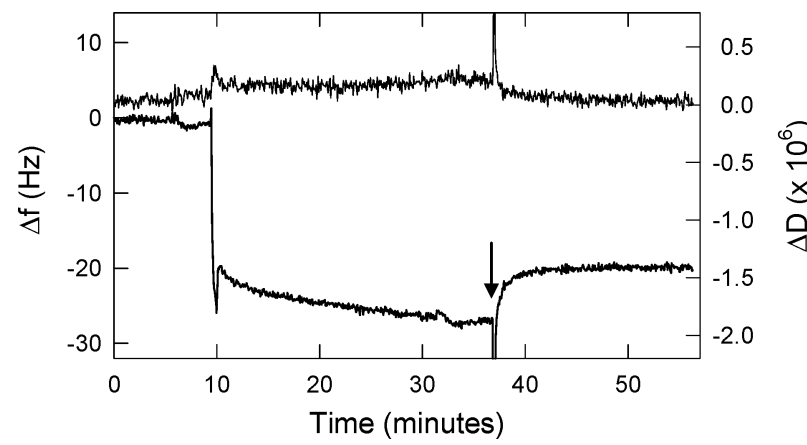

Figure 5. The adsorption of PAH-PAA polyelectrolyte complex on a lignin model film. Solution conditions: $\mathrm{pH} 7.5$ and $1 \mathrm{mM}$ $\mathrm{NaCl}$, PEC concentration $50 \mathrm{mg} \mathrm{L}^{-1}$; molar mixing ratio of the PEC, +0.3 , i.e., to 10 charge equivalents of $\mathrm{PAH}, 3$ charge equivalents of PAA are added. The arrow pointing to the frequency curve indicates Milli-Q water rinsing.

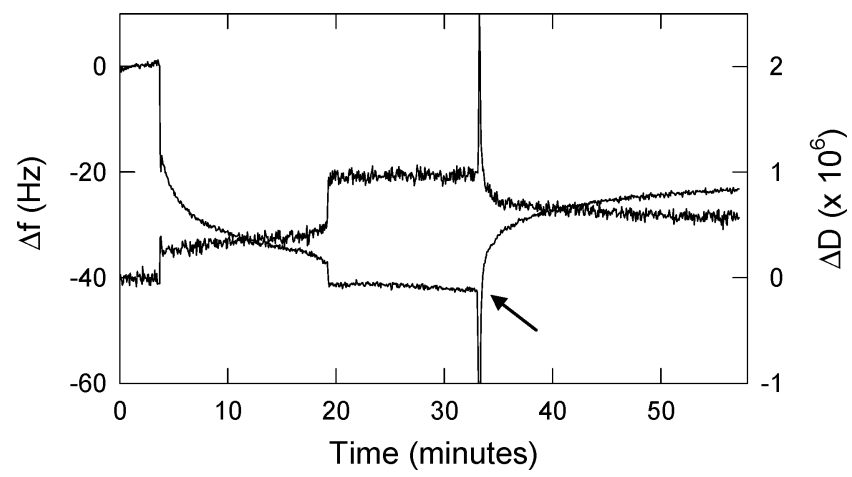

Figure 6. The adsorption of $\mathrm{PAH}$ on a lignin model surface at $\mathrm{pH}$ 7.5 and $1 \mathrm{mM} \mathrm{NaCl}$ as monitored by QCM-D. PAH concentration $50 \mathrm{mg} \mathrm{L}{ }^{-1}$. The arrow pointing to the frequency curve indicates Milli-Q water rinsing.

the experiment $\left(1.6 \mathrm{mg} \mathrm{m}^{-2}\right.$ from eq 1 including immobilized water) was at the same level as for the PEC. Moreover, a relatively flat polyelectrolyte conformation at the substrate according to the low $\Delta D$ was indicated. This is also in line with what has been reported earlier in the case of PEL adsorption at low ionic strengths. $^{45}$

A thorough investigation of the adsorption of PECs and PAH onto lignin films at various mixing ratios and at different $\mathrm{pH}-$ values and electrolyte concentrations was also made as indicated earlier. The adsorbed amount was calculated according to the Sauerbrey equation (eq 1), and a summary of the results is presented in Table 1 together with the effective hydrodynamic diameter of the PECs measured by dynamic light scattering.

From the results in Table 1 it can be seen that the highest adsorption of both the cationic PEC and PAH is obtained at the highest $\mathrm{pH}$-value ( $\mathrm{pH} 7.5)$, and mostly at the highest $\mathrm{NaCl}$ concentration $(10 \mathrm{mM})$. Moreover, at a molar mixing ratio of +0.8 and $10 \mathrm{mM} \mathrm{NaCl}$, a fairly large amount of the adsorbed PECs is removed from the lignin surface when the measuring chamber is rinsed with water. This indicates the presence of only a weak electrostatic interaction. In simple polyelectrolyte solutions, at pH 7.5 PAA can be assumed as almost fully dissociated and $\mathrm{PAH}$ is still fairly protonated, whereas at $\mathrm{pH} 5.0$ only $\mathrm{PAH}$ remains at its maximum charge. How a change of the $\mathrm{pH}$-value affects the dissociation of the polyelectrolytes in the PECs is however more complicated to tell. As can be noticed from the values in Table 1 of the effective diameters, PECs of ratio +0.3

(45) Plunkett, M. A.; Claesson, P. M.; Ernstsson, M.; Rutland, M. W. Langmuir 2003, 19, 4673.
Table 1. Summarized Data of the Adsorption of Cationic PECs and PAH on the Lignin Model Film at Various Solution Conditions (Sample Concentration $50 \mathrm{~g} \mathrm{~L} \mathrm{~L}^{-1}$ )

\begin{tabular}{ccccccc}
\hline sample $^{a}$ & $\mathrm{pH}$ & $\begin{array}{c}{[\mathrm{NaCl}]} \\
(\mathrm{mM})\end{array}$ & $\begin{array}{c}\mathrm{eff} \mathrm{diam}^{b} \\
(\mathrm{~nm})\end{array}$ & $\begin{array}{c}\Delta f_{\text {initial }}{ }^{c} \\
(\mathrm{~Hz})\end{array}$ & $\begin{array}{c}\Delta f_{\text {end }}{ }^{d} \\
(\mathrm{~Hz})\end{array}$ & $\begin{array}{c}m_{\mathrm{ads}^{e}}{ }^{2} \\
\left(\mathrm{mg} \mathrm{m}^{-2}\right)\end{array}$ \\
\hline+0.8 & 5.0 & 1.0 & 101 & -6 & -3 & 0.2 \\
+0.8 & 5.0 & 10 & 99 & -23 & -9 & 0.6 \\
+0.3 & 5.0 & 1.0 & 89 & -5 & -4 & 0.3 \\
+0.3 & 5.0 & 10 & 86 & -12 & -12 & 0.9 \\
+0.8 & 7.5 & 1.0 & 109 & -17 & -20 & 1.4 \\
+0.8 & 7.5 & 10 & 102 & -27 & -12 & 0.9 \\
+0.3 & 7.5 & 1.0 & 100 & -27 & -20 & 1.4 \\
+0.3 & 7.5 & 10 & 102 & -104 & -71 & 7.5 \\
PAH & 5.0 & 1.0 & & 0 & 0 & 0 \\
PAH & 5.0 & 10 & & -13 & 0 & 0 \\
PAH & 7.5 & 1.0 & & -42 & -23 & 1.6 \\
PAH & 7.5 & 10 & & -95 & -38 & 2.7
\end{tabular}

${ }^{a}$ In case of the PECs, +0.3 denotes highest cationic charge. ${ }^{b}$ Effective diameter of PEC determined by DLS. ${ }^{c}$ Frequency shift after adsorption. ${ }^{d}$ Final frequency shift after adsorption and water rinsing. ${ }^{e}$ Adsorbed amount together with water inclusions at final frequency shift.

(highest cationic charge) are showing a slight but not dramatic decrease in effective diameter when the $\mathrm{pH}$-value is lowered to $\mathrm{pH}$ 5. This cannot however explain the lower adsorption since PECs of ratio +0.8 also show very low adsorption at $\mathrm{pH} 5.0$, but no significant change in the effective diameter is observed. However, regarding the understanding of adsorption, it is important to take the chemistry of the substrate into account too. Recently, in a study of interaction forces between lignin and cellulose it was shown that the surface potential, and hence the surface charge, of the lignin film (identical to the films used in the present study regarding substrate and preparation method) is gradually decreasing when going from $\mathrm{pH} 8.5$ to $\mathrm{pH} 3.5 .{ }^{46}$ From the obtained force-distance curves at $0.1 \mathrm{mM} \mathrm{NaCl}$, the lignin film surface potential was determined to be $-75 \mathrm{mV}$ at $\mathrm{pH} 8.5,-60 \mathrm{mV}$ at $\mathrm{pH} 5.8$, and $-5 \mathrm{mV}$ at $\mathrm{pH} 3.5$ according to the Grahame equation. ${ }^{47}$ This change was in good agreement with the results obtained from theoretical modeling of the lignin film by applying the Poisson-Boltzmann cell model ${ }^{48,49}$ in planar symmetry based on literature data of the contents of carboxylic and phenolic groups in kraft lignin and the $\mathrm{p} K_{\mathrm{a}}$-values of these functional groups. Thus, the electrostatic contribution from the negatively charged lignin model surface to the interaction forces is stronger at higher $\mathrm{pH}$-values, decreases by increasing ionic strength at a given $\mathrm{pH}$ value, and is mainly due to an increase in the dissociation of carboxylic groups at $\mathrm{pH}$ values below 9.5. ${ }^{46}$ This seems also to be the best explanation of the stronger attraction of cationic $\mathrm{PEC}$ and $\mathrm{PAH}$ to the lignin film at $\mathrm{pH} 7.5$ than at $\mathrm{pH} 5.0$.

Adsorption of PAA and Anionic PECs. Concerning the fact that the lignin film is slightly negatively charged above $\mathrm{pH} 3.5$ due to the dissociation of some few carboxylic acid groups in the kraft lignin macromolecular structure, ${ }^{46}$ the result presented in Figure 7 is perhaps somewhat unexpected. Figure 7 shows that at $10 \mathrm{mM}$ and $\mathrm{pH} 7.5$ the adsorption of PAA on a lignin coated crystal is very high in comparison to what is found for PAH and cationic PECs on the same substrate. From the Sauerbrey equation (eq 1), the adsorbed amount of PAA together with the accompanying solvent corresponds to a surface coverage of 39 $\mathrm{mg} \mathrm{m}^{-2}$. Earlier, XPS measurements performed by Plunkett et al. showed that the polyelectrolyte charge density plays an important role on the frequency shift of the QCM-D crystal during adsorption. ${ }^{45}$ Whereas XPS only gives the absolute amount of

(46) Notley, S. M.; Norgren, M. Langmuir 2006, 22, 11199.

(47) Israelachvili, J. Intermolecular and surface forces, 2nd ed.; Academic Press: New York, 1991.

(48) Gunnarsson, G.; Jönsson, B.; Wennerström, H. J. Phys. Chem. 1980, 84, 3114.

(49) Norgren, M.; Lindström, B. Holtzforschung 2000, 54, 519. 


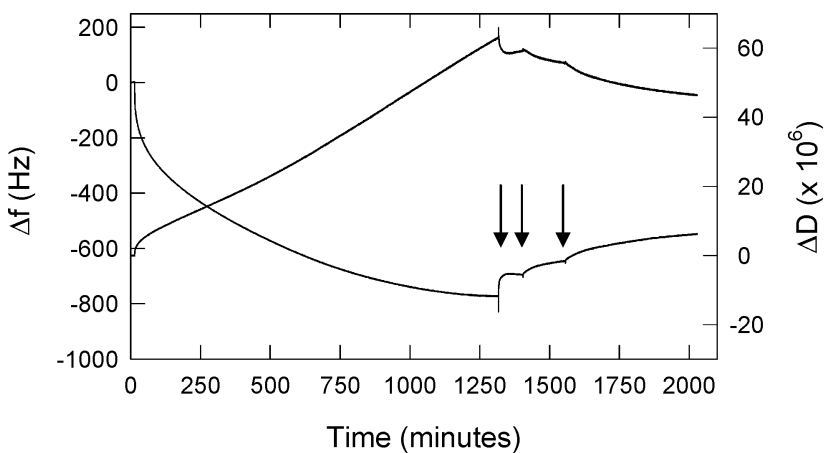

Figure 7. The adsorption of PAA on a lignin film at $10 \mathrm{mM} \mathrm{NaCl}$ and $\mathrm{pH} 7.5$ as monitored by QCM-D. PAA concentration $50 \mathrm{mg}$ $\mathrm{L}^{-1}$. The arrows pointing to the frequency curve indicate the time of Milli-Q water rinsing of the sample chamber.

adsorbed polyelectrolyte, the frequency shift determined from QCM-D measurements also includes solvent incorporated in the adsorbed layer. According to XPS measurements, the adsorbed amount was found to decrease in a more or less linear manner from about $2.5 \mathrm{mg} \mathrm{m}^{-2}$ to approximately $1 \mathrm{mg} \mathrm{m}^{-2}$ as the charge of the polyelectrolyte increased from $1 \%$ to $100 \%$. $\Delta f$, on the other hand, showed a more nonlinear behavior. In the polyelectrolyte charge range $1 \%$ to $100 \%$, the corresponding adsorption according to the Sauerbrey equation (eq 1) decreased from $11 \mathrm{mg} \mathrm{m}^{-2}$ to $0.8 \mathrm{mg} \mathrm{m}^{-2}$. The differences in the results between the two methods were explained to be attributed by the specific conformation that is adopted by the polyelectrolytes at the surface, giving rise to an adsorbed-layer-solvent-content of approximately $80 \%$ at a charge level of $1 \%$ down to almost zero at $100 \%$ polyelectrolyte charge. In a recently published study, Enarsson showed that in case of the adsorption of cationic polyacrylamide and cationic dextran on silica, a water fraction of around $80 \%$ can be expected at an electrolyte concentration of $10 \mathrm{mM} .{ }^{50}$ Taking the inclusion of water into account, the adsorbed amount of pure PAA would be approximately $8 \mathrm{mg}$ $\mathrm{m}^{-2}$ if the outcome of the latter study would be directly transferred to this system. However, this indicates a high amount of adsorbed polymer that is not possible to obtain on a flat nonporous surface, due to geometrical packing reasons. Thus, with respect to this and by considering the very high measured final change in dissipation, $\Delta D=47 \times 10^{-6}$, it must be concluded that PAA is very weakly attached to the lignin film and $80 \%$ inclusion water in the adsorbed PAA layer is clearly an underestimation in the present system.

In Figure 8, a QCM-D plot of the adsorption of an anionic PAA-PAH PEC with a molar mixing ratio of -0.8 can be seen. DLS measurements revealed an effective diameter of $138 \mathrm{~nm}$ directly after the preparation of the anionic PEC, which after addition of $\mathrm{NaCl}$ to a sample concentration of $10 \mathrm{mM}$ and $\mathrm{pH}$ adjustment to 7.5 increased to $341 \mathrm{~nm}$ in the dispersion used for the adsorption experiment. Comparably larger sizes of anionic PECs than cationic PECs prepared from the present polyelectrolytes have been observed earlier after PEC preparation with the reversed mixing order of the polyelectrolytes. ${ }^{51}$ This phenomenon is rather system specific and depends more on the physical and chemical characteristics of the polyelectrolytes (chain stiffness, functional groups, etc.) rather than the molecular weight. ${ }^{36}$ Generally, an increase in the effective diameter is partly

(50) Enarsson, L.-E. Licentiate thesis. Polyelectrolyte adsorption on oppositely charged surfaces. Conformation and adsorption kinetics. Royal Institute of Technology, Stockholm, 2006.

(51) Gärdlund, L. Doctoral thesis. Polyelectrolyte complexes. Their characterisation and use for modification of wood fibre surfaces. Royal Institute of Technology, Stockholm, 2006.

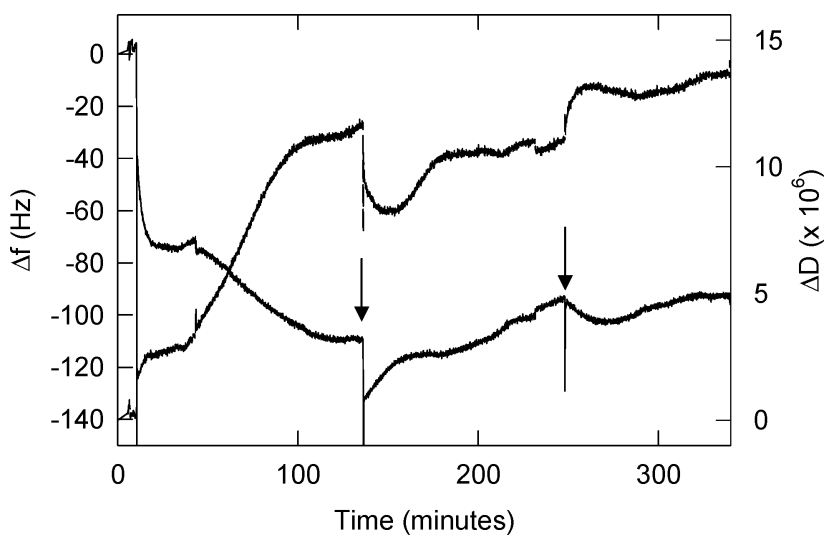

Figure 8. QCM-D data from the adsorption of an anionic PAAPAH PEC on a lignin model film. Solution conditions: $\mathrm{pH} 7.5,10$ $\mathrm{mM} \mathrm{NaCl}, \mathrm{PEC}$ concentration $50 \mathrm{mg} \mathrm{L}^{-1}$; molar mixing ratio of the $\mathrm{PEC},-0.8$. The arrows pointing to the frequency curve indicate when Milli-Q water rinsing was performed.

due to the weakening of the Columbic interactions between the anionic and cationic groups by salt screening, giving rise to an internal network with fewer cross-linked nodes, which governs the swelling. In the present case a slight aggregation of the anionic PEC particles could probably be attributed.

In the adsorption of anionic PECs, the $\mathrm{pH}$ and ionic strength were held at the same levels as in the PAA adsorption experiment shown in Figure 7. After about $140 \mathrm{~min}$, the adsorption of the anionic PEC declined and the measuring chamber was rinsed with Milli-Q water. As is seen from the change in dissipation, the rinsing induces an initial reduction in the viscosity of the adsorbed layer, indicating conformational change of the PECs and deswelling. After the first rinsing, gradually some of the PECs also desorbs from the surface. At about $250 \mathrm{~min}$, a second water rinsing was performed and an increased swelling of the adsorbed film can be detected as an increase in dissipation and a small decrease in frequency.

The lignin-coated QCM-D crystal from the adsorption experiment presented in Figure 8 was air-dried during $48 \mathrm{~h}$ in a laminar flow cabinet and further analyzed by AFM in tapping-mode. Already during the setup of the AFM experiment, regions where extensive adsorption of PEC aggregates had occurred could be spotted by the optical microscope. To avoid contamination of the tip, the AFM imaging was performed where no macroscopic aggregates could be detected. The outcome of the analysis revealed that the anionic PECs adsorbed exist as caplike particles on the lignin surface after air-drying. Figure 9 is a schematic illustration showing AFM images of the lignin film on a QCM-D crystal before and after PEC adsorption, and how the PECs appear in solution, as was caught by cryogenic transmission electron microscopy (cryo-TEM).

Adsorption of negatively charged polyelectrolytes on a slightly negative substrate is however not unique at medium to high electrolyte concentrations due to a decreased electrostatic repulsion between the polyelectrolyte and the substrate surface..$^{52}$ The nonionic interactions between the polyelectrolyte and the surface could assist the polyelectrolyte to overcome the repulsive barrier and adsorb to the surface. The strength of the nonionic interaction is generally system specific and thus dependent on both the characteristics of the polyelectrolyte and the chemistry of the substrate. ${ }^{52,53}$ Moreover, a high electrolyte concentration

(52) Fleer, G. J.; Cohen-Stuart, M. A.; Scheutjens, J. M. H. M. In Polymers at Interface; Cosgrove, T., Vincent, B., Eds.; Chapman \& Hall: London, 1993.

(53) van de Steeg, H. G. M.; Cohen-Stuart, M. A.; de Keizer, A.; Bijsterbosch, B. H. Langmuir 1992, 8, 2538 


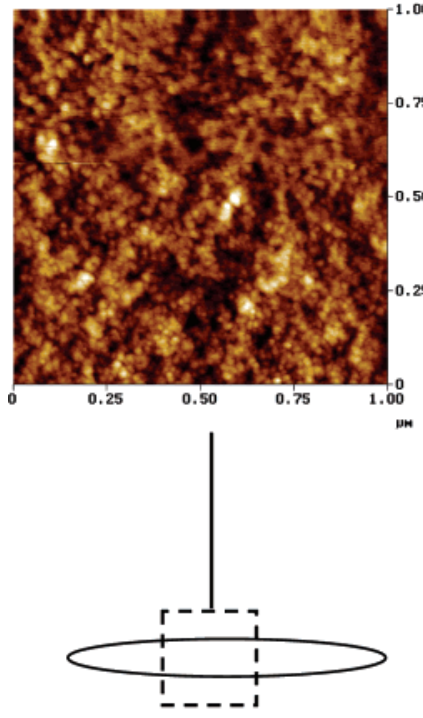

lignin-coated qcm crystal

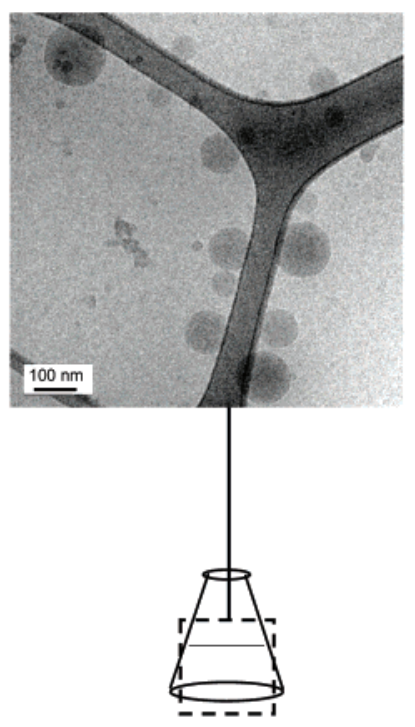

PECs in solution

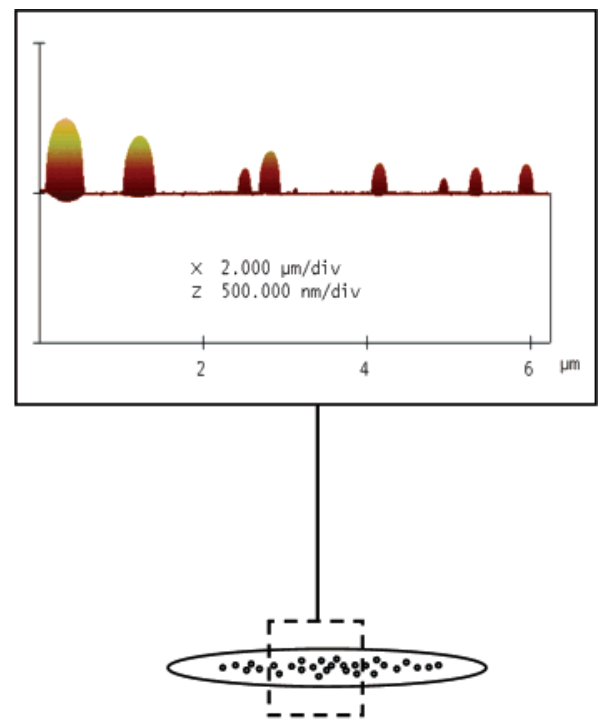

anionic PECs adsorbed

Figure 9. Schematic illustration of the appearance of (a, left) the lignin film on a QCM-D crystal imaged by AFM, (b, middle) PECs in solution as caught by cryo-TEM, and (c, right) anionic PAA-PAH PECs adsorbed on a lignin-coated QCM crystal as imaged by AFM.

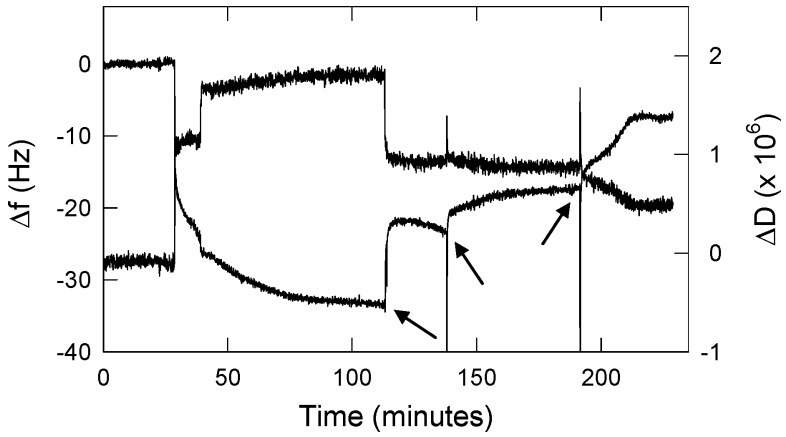

Figure 10. Adsorption of PAA on silica at $100 \mathrm{mM} \mathrm{NaCl}$ and $\mathrm{pH}$ 7.5. The PAA concentration was $50 \mathrm{mg} \mathrm{L}^{-1}$. The arrows pointing to the frequency curve indicate when Milli-Q water rinsing was performed.

could also induce salting-out effects, i.e., decrease the polymer solubility and thereby cause the polymer to precipitate onto the substrate surface. This latter phenomenon is usually relatively independent of the substrate chemistry.

Salt Induced Adsorption of PAA Due to Decreased Polyelectrolyte Solubility? In an effort to investigate what is causing the very high adsorption of PAA on lignin model surfaces, the idea of enhanced adsorption due to reduced polyion solubility at higher ionic strengths was tested. Uncoated, silica sputtered QCM crystals were used, and the adsorption of $50 \mathrm{mg} \mathrm{L}^{-1}$ PAA solutions containing $10 \mathrm{mM}$ and $100 \mathrm{mM} \mathrm{NaCl}$, respectively, was investigated. At a concentration of $10 \mathrm{mM} \mathrm{NaCl}$, the final frequency shift after rinsing with water reached $\Delta f_{\text {end }}=-8 \mathrm{~Hz}$ and the change in dissipation was $0.5 \times 10^{-6}$. From using eq 1 , this corresponds to an adsorbed amount of about $0.6 \mathrm{mg} \mathrm{m}^{-2}$ with water included, which is less than one-tenth of what was adsorbed on the lignin film at the same experimental conditions. In Figure 10 the adsorption of PAA at a concentration of 100 $\mathrm{mM} \mathrm{NaCl}$ is depicted. Here the initial adsorption before rinsing with water gives rise to a frequency shift of around $-35 \mathrm{~Hz}$ after equilibrating the system. At the same time the dissipation increases, indicating spatial extension of an adsorbed PAA layer on the crystal. However, after three rinses with water $\Delta f_{\text {end }}$ reaches $-8 \mathrm{~Hz}$ and the change in dissipation goes down to $0.5 \times 10^{-6}$. This is equivalent to what was determined for the adsorption of
PAA on silica in $10 \mathrm{mM}$ sodium chloride. Obviously, the strength of the attractive interaction between the silica surface and PAA is of a much lower magnitude than what is seen between PAA and the lignin film.

Moreover, this also points to that an increased adsorption due to a supposed reduction of the polyion solubility is not the case for PAA and cannot explain the high affinity to the lignin film that is observed. Thus, the extensive adsorption to lignin model surfaces found both for PAA and the anionic PAA-PAH polyelectrolyte complex indicates the presence of strong nonionic interactions in these systems.

\section{Conclusions}

Kraft lignin model films prepared according to a recently developed protocol were used successfully in polyelectrolyte adsorption studies. This brings about new possibilities to achieve important information about the physicochemical characteristics of lignin in solid state on a molecular level. From the outcome of the experiments it was further concluded that electrostatic interactions between the lignin model surfaces and polyallylamine or cationic PECs investigated were important. This was attributed by an increased adsorption when the $\mathrm{pH}$-value of the solution was increased and the dissociation of carboxylic groups on the lignin surface increased. However, electrostatic interactions are not the only driving force of adsorption. Poly(acrylic acid) as well as anionic PECs showed a specific affinity toward the lignin film and were adsorbed to comparably high amounts. In the latter cases, nonionic interactions between poly(acrylic acid) and lignin are suggested to be the reason for the extensive adsorption observed.

Acknowledgment. L.G. gratefully acknowledges the Kempe Foundation for economic support. S.M.N. would like to acknowledge financial support from the Co-operative Research Centre for Functional Communication Surfaces (CRC SmartPrint). Gunnel Karlsson, the Biomicroscopy Unit at Lund University, is acknowledged for invaluable assistance in the cryoTEM analysis. Göran Gellerstedt and Andrea Majtnerova, Royal Institute of Technology, Stockholm, are thanked for the isolation and purification of the kraft lignin.

LA063439Z 